Original Research Article

\title{
Pattern of antimicrobial utilization in indoor ward of surgery department of a tertiary care teaching hospital of Southern Rajasthan, India
}

\author{
Vinod Kumar Chhipa, Meena Atray*
}

Department of Pharmacology, Rabindranath Tagore Medical College (R.N.T.), Udaipur, Rajasthan, India Received: 26 April 2017
Accepted: 24 May 2017

\section{*Correspondence to:}

Dr. Meena Atray, Email: drmatray@gmail.com

Copyright: (C) the author(s), publisher and licensee Medip Academy. This is an openaccess article distributed under the terms of the Creative Commons Attribution NonCommercial License, which permits unrestricted noncommercial use, distribution, and reproduction in any medium, provided the original work is properly cited.

\begin{abstract}
Background: Drug utilization studies are conducted to analyze prescription pattern, facilitate rational prescribing and improve the outcome. Antibiotics are frequently prescribed drugs in surgery department both by oral and parenteral route. Irrational prescribing of antibiotics is responsible for emergence of drug resistance in pathogens which is a global problem now a days.

Methods: 225 patients admitted in surgical indoor were included in the study and data collected was evaluated as per WHO quality indicators.

Results: Ceftriaxone was maximally utilized antimicrobial (\%), average number of antimicrobial prescribed was $2.73 \pm 1.08$. Average duration of hospitalization was 10.32 days. Costliest antimicrobial prescribed was meropenum and with minimum cost was Clarithromycin. Cost per patient was Rs.72.6. PDD/DDD ratio of Amoxicillin+Clavulanic acid was maximum. 24\% of patients were receiving complementary and alternative medicines.

Conclusions: The drugs were prescribed from the essential drug list with generic names in appropriate dose and duration. The need of Culture and sensitivity test for selecting antimicrobials was identified in the study. The study can be further expanded by including patients of vulnerable groups and private sector and evaluation of effect of self medication on outcome.
\end{abstract}

Keywords: Antimicrobial utilization, Prescription pattern, Surgery indoor

\section{INTRODUCTION}

Drug utilization research was defined by WHO in 1997 , as "the studies of marketing, distribution, prescription and use of drugs in a society with special emphasis on the resulting medical, social and economic consequences. ${ }^{1,2}$

Drug utilization studies are helpful in analyzing prescription pattern, to calculate adverse drug reaction, to estimate disease prevalence and to estimate drug expenditures. It facilitates rational use of drugs with improved outcome. ${ }^{3}$ Antibiotics deserve an important place in improving life expectancy of human beings by preventing and treating infectious diseases. According to official circular issued by CDSCO in 2012, antibiotics are prescribed unnecessary in $70-80 \%$ of prescriptions by healthcare providers, which indicate need of strict regulatory instructions for prescribing antibiotics as scarcity of newer antibiotics is a big emerging global problem. ${ }^{4}$

Rational prescribing for antibiotics is of great importance as overuse of antibiotics may cause unnecessary adverse effects and it also increases cost of therapy while underuse and unnecessary use of antibiotics may cause development of resistance. ${ }^{4,5}$ Rational use of drugs means prescribing the drug in appropriate dose and for appropriate duration according to clinical need of patient at affordable price. In developing countries rational prescribing is also important for optimal utilization of health budget as irrational prescribing may result in scarcity of essential medicine. ${ }^{5-7}$ 
Patients admitted in surgery indoor need surgical intervention and intensive drug therapy. Intravenous analgesics and antimicrobials are frequently used and in most of the cases their use is justifiable but it needs frequent review, proper guidelines and monitoring. ${ }^{8-11}$

With this background, this study was designed to analyze the prescription pattern in a tertiary care teaching hospital, to evaluate the collected data as per WHO quality indicators, to analyze cost of prescribed antimicrobials and provide feedback to the prescribers for continuous improvement.

\section{METHODS}

After taking consent from institutional ethics committee and Head of the Department of Surgery, this prospective cross-sectional study was conducted in indoor of surgery department for duration of six months from January 2016 to June 2016.

225 patients admitted in male and female indoor fulfilling inclusion criteria were included in the study.

\section{Inclusion criteria}

- Patients admitted in male and female surgery ward.

\section{Exclusion criteria}

- Patients with severe ailments, shifted to ICU from indoor.

- Pregnant women

- Patients who deny to participate.

A standard subject socio demographic and clinical features data collection proforma was prepared and information regarding age, gender, occupation, income group, subject IP/OP number, diagnosis, subject present/past medical history, number of days of hospitalization, treatment, any ADR, investigations, outcome, Patients knowledge about drug treatment, selfmedication was collected from indoor ticket and patient's interview.

Antimicrobial utilization pattern among male and female patients was evaluated using quality indicators of drug use, recommended by WHO. Ailments for which antimicrobials were prescribed, Number of antimicrobials prescribed, route of administration, any adverse drug reaction occurred during treatment were recorded. Average number of antimicrobials per prescription, Percentage of antimicrobials prescribed by generic name, percentage of antimicrobials prescribed from essential drug list, average dose of antimicrobial per prescription, PDD/DDD ratio, cost of antimicrobials prescribed, average cost per prescription, total burden on government were calculated.
Data was statistically analyzed by entering it into a Microsoft excel sheet

\section{RESULTS}

Out of 225 patients included, $126(56 \%)$ were male and $99(44 \%)$ were female patients.

Clinical diagnosis for which antimicrobials were prescribed among surgical indoor patients were GITdisorder $81(36 \%)$, Genito-urinary disorders $36(16 \%)$, trauma 21 (9.33), Carcinoma 18 (8\%), Lump 18 (8\%), Plastic surgery 15 (6.67\%), Hernia 12 (5.33\%), Gangrene $9(4 \%)$, Liver disease $6(2.67 \%)$, Cellulites $3(1.33 \%)$, Cyst 3 (1.33\%), Sarcoma 3 (1.33\%) (Figure 1).

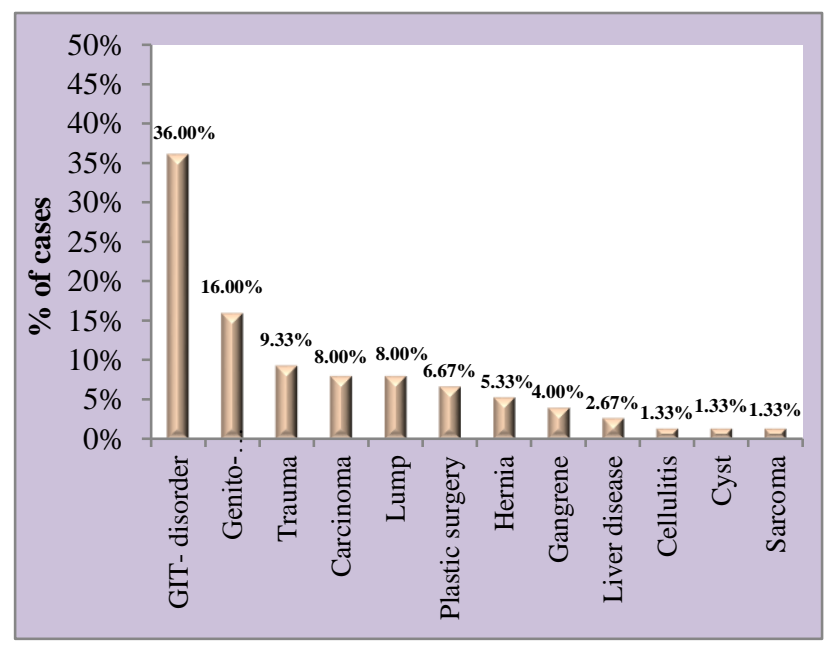

Figure 1: Clinical diagnosis of patients admitted in surgical indoor.

Total number of antimicrobials used was 13. Ceftriaxone was the maximally utilized antimicrobial, given to 195 patients. Mean number of antimicrobials prescribed per patient was $2.73 \pm 1.08$.

Total number of antimicrobials administered by IV route was $495(80.49 \%)$, and by oral route $120(19.51 \%)$ costliest antimicrobial prescribed was meropenam, prescribed to 12 patients. Total cost per patient for meropenam was Rs.4566.76. In terms of cost, cheapest antimicrobial was clarithromycin oral, given to 3 patients. Cost per patient was Rs.8.8. Cost of all the antimicrobials utilized was of Rs.168577.40. Cost per patient was Rs. 749.23 and cost per patient per day was Rs. 72.6.

All antimicrobials prescribed in the institution are free supply from the Government. So treatment causes no financial burden to the patient.

Prescribed daily dose of antimicrobials were compared with defined daily dose. PDD/DDD ratio of amoxicilline+clavulanic acid oral was maximum (1.87) and amoxicilline+clavulanic IV was minimum (0.46). 
In surgical indoor 54 (24\%) patients were receiving CAM (complementary and alternative medicines) other than antimicrobials.

No severe ADR was reported during the study ADRs reported were headache $(28 \%)$, nausea $(26 \%)$, vomiting $(11 \%)$ and rashes $(2 \%)$.

Table 1: Cost analysis of prescribed antimicrobials.

\begin{tabular}{|llll|}
\hline Antimicrobial & $\begin{array}{l}\text { No. of } \\
\text { patients }\end{array}$ & $\%$ & $\begin{array}{l}\text { Total cost of } \\
\text { antimicrobial }\end{array}$ \\
\hline Meropenam & 12 & 1.95 & 54801.2 \\
\hline Amikacin & 147 & 23.90 & 32661.0 \\
\hline Ceftriaxone & 195 & 31.71 & 31073.7 \\
\hline Ceftazidime & 15 & 2.44 & 16377.4 \\
\hline Metronidazole & 111 & 18.05 & 14981.1 \\
\hline $\begin{array}{l}\text { Pipracillin+ } \\
\text { Tazobactum }\end{array}$ & 9 & 1.46 & 10597.0 \\
\hline Linezolid & 3 & 0.49 & 2478.6 \\
\hline $\begin{array}{l}\text { Amoxicilline+ } \\
\text { Clavulanic acid } \\
\text { oral }\end{array}$ & 45 & 7.32 & 1865.6 \\
\hline Cefixime & 54 & 8.78 & 1365.5 \\
\hline $\begin{array}{l}\text { Amoxicilline+ } \\
\text { Clavulanic acid } \\
\text { IV }\end{array}$ & 6 & 0.98 & 1226.3 \\
\hline Ciprofloxacine & 9 & 1.46 & 972.0 \\
\hline Ofloxacin & 3 & 0.49 & 117.0 \\
\hline Levofloxacin & 3 & 0.49 & 34.5 \\
\hline Clarithromycin & 3 & 0.49 & 26.4 \\
\hline Total cost & & & 168577 \\
\hline Cost per patient & & & 749.23 \\
\hline Cost per day & & & 72.6 \\
\hline
\end{tabular}

\section{DISCUSSION}

Aim of drug utilization studies is to analyze and evaluate prescribing pattern by using standard drug use indicators, and suggest modifications if needed to the prescribers for making the treatment more rational and cost effective. ${ }^{12}$

Irrational use of antimicrobials may increase cost of treatment, cause more ADRs and higher rate of antimicrobial resistance in community pathogens.

The study was planned to analyze and evaluate prescribing pattern of antimicrobials in a tertiary care teaching hospital, to compare it with other studies and to provide feedback to the prescribers.

Our study has covered population from rural as well as urban areas, different socio economic and age groups. There were very few studies available to compare the results.

Mean duration of hospital stay was 10.32 days. In study by Rasheeduddin et al, it was 9.2, which was very near to our studywhile in study by Teferra Abula and Mohammed Kedir, it was 14.2, for which selection of serious patients in their study might be the reason. ${ }^{8,13}$

Total number of drugs administered by IV route was 495 $(80.49 \%)$ and by oral route $120(19.51 \%)$. Nearly similar results were reported in study by Rasheeduddin et al.

Generally IV routes are preferred in surgical patients, as drugs by IV route attain required blood concentration which is usually required in surgical procedures.

Ceftriaxone was the maximally utilized antimicrobial, given to 195 patients. (Table 1). The pattern reported in study by Rasheeduddin was very near to our study but in comparison to them flouroquinolol was less utilized in our study, while in study by Taferra Abdulla, Mohammed Kedir, ampicillin, gentamycin and chloramphenicol were used. In study by Mondal et al, gentamycin was used in place of amikacin in our study. 8,14

Mean number of antimicrobials prescribed per patient was $2.73 \pm 1.08$. In study by Taferra Abdulla, Mohammed Kedir, it was 2.17 indicating overutilization of antimicrobials in our study. Though their study was done in 2004, and the difference may be due to changing sensitivity and resistance among pathogens. In other studies, average number of antimicrobials per patient was not reported.

All antimicrobials prescribed in the institution are free supply from the Government. So treatment causes no financial burden to the patient. However in our study financial burden to the government and average cost per patient per day was calculated.

In surgical indoor patients, costliest antimicrobial prescribed was meropenam, prescribed to 12 patients. Total cost per patient for meropenam was Rs.4566.76. antimicrobial with minimum cost was clarithromycin, given to 3 patients. Cost per patient was Rs.8.8. Total cost of all the antimicrobials utilized was of Rs.168577.40. Cost per patient was Rs. 749.23 and cost per patient per day was Rs. 72.6 (Table 1). Study by Shelat et al, was conducted in private hospitals and the cost per patient was very high due to use of branded drugs while in our institution, drugs are prescribed by generic names and purchased by government which are provided to every patient free of cost. ${ }^{15}$ In developing countries like India it helps in providing health facilities to maximum number of patients in limited cost. This information strongly indicates the need for comparative evaluation of cost in private and government sectors, comparison of pharmacokinetic parameters and accordingly establishment of policy to prescribe antimicrobials

Appropriate dose and duration is one of the major determinants to control development of bacterial resistance. Most of the drugs were prescribed in 
appropriate dose. For amoxicillin-clavulanic acid oral, PDD/DDD ratio was 1.87 and for IV it was 0.46. As DDD is the dose calculated for maintenance therapy and in indoor most of the admitted patients need aggressive treatment. This may be the reason for increased PDD/DDD ratio. Few antimicrobials

were started empirically and stopped after one or two doses, because the therapy was changed after final diagnosis. This was the probable cause for low PDD/DDD ratio.

In surgical indoor 54 (24\%) patients were receiving CAM (complementary and alternative medicines) other than antimicrobial. CAM may affect the course of disease or effect of antimicrobials, may interact with antimicrobials and increase occurrence of ADRs. This finding indicates role of household remedies, self-medication and role of quacks in our country. People in village, illiterate patients and patients from low socioeconomic status try to treat the disease at home first and then they come to hospital. It also indicates need of free drug supply at government hospitals.

In the institute, all drugs were provided free of cost and most of the groups were available, the drugs were prescribed with generic names and from the essential drug list. No irrational drug combination of antimicrobial was prescribed. Most of the drugs were appropriately used.

Culture sensitivity test was not done for selection of antimicrobials in most of the cases and antimicrobials were prescribed empirically.

The study included limited number of patients who were not be followed up after discharge. The individual variation in choice of treatment can also affect the results and it could not be included in the study. So it could not evaluate generalized pattern in the institution. The drugs available were also limited and comparison of cost of different brands could not be possible. Involvement of CAM was found but could not be analyzed. Very few studies were available to compare the results.

The study can further be expanded in future including different clinical departments, antimicrobial utilization in vulnerable groups including pregnant and paediatric patients, effect of CAM in therapy, comparison of drug utilization in government and private sectors to promote rational use of drugs and maximum utilization of resources.

\section{CONCLUSION}

The present study was undertaken to study and evaluate the pattern of antimicrobial utilization by using $\mathrm{WHO} /$ INRUD indicators in surgical indoor patients in relation to pharmacoepidemiology, pharmacovigilance and pharmacoeconomics.
The study indicates various encouraging findings like the antimicrobials were prescribed from essential drug list, by generic names and free of cost to the patients. PDD/DDD ratio of most of the antimicrobials was appropriate except amoxicillin-clavulanic acid IV (0.46) and oral (1.87).

Study indicate need of culture sensitivity test for prescribing antimicrobials.

Limited number of patients and available drugs were the major limiting factors. The study can be further expended by including other departments, private sector and vulnerable group of population. The study can also include effect of CAM.

The results and feedback provided by the study would be helpful in constructing rules and regulations for drug utilization at institutional level.

\section{ACKNOWLEDGMENTS}

Authors would like to thank faculty and residents of IV Unit of Surgery Department.

Funding: No funding sources Conflict of interest: None declared

Ethical approval: The study was approved by the Institutional Ethics Committee

\section{REFERENCES}

1. WHO Expert Committee Report, The Use of Essential Drug, Technical Report Series NO. 770, Geneva, World Health Organization, 1998.

2. Sachdeva PD, Patel BG. Drug utilization studiesscope and future perspectives. Int J Pharm Biol Res. 2010;1:11-7.

3. Shalini S, Ravichandran V, Mohanty BK, Dhanaraj SK, Saraswathi R. Drug utilization studies-An overview. Int J Pharm Sci Nanotechnol. 2010 Apr;3(1):803-10.

4. Paterson DL, Rogers BA. How soon is now? The urgent need for randomized, controlled trials evaluating treatment of multidrug-resistant bacterial infection. Clin Infect Dis. 2010;51:1245-7.

5. Lockhart SR, Abramson MA, Beekman SE, Gallagher G, Riedel SR, Diekma DJ, et al. Antimicrobial resistance among gram negative bacilli as causes of infection in intensive care unit patients in the united states between 1993 and 2004. J Clin Microbiol. 2007;45:3352-9.

6. Badar VA, Navale SB. Study of prescribing pattern of antimicrobial agents in medicine intensive care unit of a teaching hospital in Central India. JAPI. 2012;60:20-3.

7. The rational use of drugs, report on the conference of experts Nairobi sponsored by WHO Geneva; 1985:25-29. 
8. Abula T, Kedir M. The pattern of antibiotic usage in surgical in-patients of a teaching hospital, northwest Ethiopia. Ethiop. J. Health Dev. 2004;18(1).

9. Dashputra AV, Badwaik RT. Utilization of analgesics in perioperative cases of teaching hospital. Int J Med Pharm Sci. 2013;3:14-9.

10. Gupta N, Sharma D, Garg SK, Bhargava VK. Auditing of prescriptions to study utilization of antimicrobials in a tertiary hospital. Indian $\mathbf{J}$ Pharmacol. 1997;29:411-5.

11. Kass EH. Antimicrobial drug usage in general hospitals in Pennsylvania. Ann Int Med. 1978;89:800-80.

12. Paterson DL. The role of antimicrobial management programs in optimizing antibiotic prescribing within hospitals. Clinical Infectious Diseases 2006;42:90-5.

13. Mohd R, Ravi Shankar B, Babu JN, Reddy NL. Antibiotic Utilization Study in the Department of Surgery of a Teaching Hospital and Research Centre. Scholars Journal of Applied Medical Sciences
(SJAMS). Sch. J. App. Med. Sci. 2016;4(8C):291823.

14. Monall S, Sushobhan P, Mallick B, Sengupta M, Niyogi S, Chaudhuri PR. A Drug Utilization Study in the Indoor Ward of the Surgery Department of a Tertiary Care Hospital of Eastern India. IOSR Journal of Dental and Medical Sciences (IOSRJDMS) e-ISSN: 2279-0853, p-ISSN: 2279-0861. 2015;14(10):42-7.

15. Shelat PR, Kumbar SK. A Retrospective Analysis of Direct Medical Cost and Cost of Drug Therapy in Hospitalized Patients at Private Hospital in Western India. Journal of clinical and diagnostic research: JCDR. 2015 Nov;9(11):FC09.

Cite this article as: Chhipa VK, Atray M. Pattern of antimicrobial utilization in indoor ward of surgery department of a tertiary care teaching hospital of Southern Rajasthan, India. Int J Basic Clin Pharmacol 2017;6:1723-7. 